apoptosis and autophagy. Reduced cathepsin B and D with increased cathepsin $\mathrm{L}$ is a phenotype suggesting reduced autophagy. Dysregulation of autophagy may be important in the pathogenesis of APS. Therefore, we aimed to determine the effect of aPL on monocyte cathepsin balance and autophagy.

Methods Healthy control (HC) monocytes were treated with $200 \mu \mathrm{g} / \mathrm{ml}$ of $\operatorname{IgG}$ purified from $(\mathrm{n}=9)$ patients with APS-IgG or $(n=9)$ HC-IgG for 6 hour. Cathepsin B and D expression were measured by western blot. Cathepsin D, B and L activity were measured using fluorescence-based assays. Intracellular proteolytic activity was determined using DQ-BSA.

Results Consistent with our previous proteomic analysis, western blots confirmed that cathepsin B and cathepsin D were down-regulated in monocytes treated with APS-IgG compared to HC-IgG. Similarly, cathepsin B and D activities were significantly reduced in monocytes treated with APS-IgG ( $p=0.0188$ for $\mathrm{B}, 0.0323$ for $\mathrm{D})$. In contrast, cathepsin L activity was increased in monocytes treated with APS-IgG $(p=0.0106)$. We then examined cathepsin activity in monocytes from patients with APS. Cathepsin L activity was increased significantly when these monocytes were treated with APS-IgG compared to $\mathrm{HC}-\operatorname{IgG}(\mathrm{p}=0.0257)$.

Lysosomal proteolysis is a key process in the late phase of autophagy. We therefore exposed $\mathrm{HC}$ monocytes to $\mathrm{IgG}$, treated them with GM-CSF overnight and tested their intracellular proteolytic activity. APS-IgG reduced the lysosomal activity of GM-CSF-treated monocytes whereas HC-IgG had no effect.

Conclusions We found that APS-IgG regulate the expression and activity of lysosomal proteases cathepsins $\mathrm{B} / \mathrm{D}$ and cathepsin L in opposite directions. Consistent with this finding, APS-IgG reduced lysosomal proteolysis in monocytes. Additional experiments are now underway to increase our understanding of how modulation of cathepsin activity and autophagy may be important in the pathogenesis of APS and to provide new therapeutic targets.

\section{S6D:6 INHIBITION OF EIF4A TRANSLATION INITIATION AMELIORATES LUPUS MANIFESTATIONS}

M Morell, G Gómez-Hernández, N Varela, M Alarcón-Riquelme. Centro de Genómica e Investigación Oncológica: Pfizer/Universidad de Granada/Junta de Andalucía, Granada, Spain

\subsection{6/lupus-2018-abstract.39}

Systemic Lupus Erythematosus (SLE) is an autoimmune disease characterised by loss of tolerance and presence of auto reactive $\mathrm{B}$ and $\mathrm{T}$ cells. Pathogenic mechanisms of the disease are still unclear and clinical manifestations are heterogeneous complicating diagnosis and disease treatment. Several organs can be affected such as skin, joints, central nervous system, and kidneys. Current treatments include corticosteroids and immunosuppressive drugs, nonsteroidal anti-inflammatory drugs, antimalarials and other biologicals such as belimumab or rituximab. During the last decades mouse models have been very helpful to understand lupus pathogenesis and to test the efficacy of SLE therapies. In this work we evaluated the therapeutic potential of inhibiting the translation initiation process with Pateamine A, a natural compound isolated from a marine sponge, in a lupus preclinical model. Translation initiation via the eIF4E complex is a key step in cytokine and antibody production, and its inhibition has been successful in cancer models. Pateamine A has anti-proliferative and immunosuppressive activities. In the BXSB.Yaa strain, we tested the efficiency of Pateamine A in controlling lupus autoimmune symptoms and neurological complications. Animal treatment started when first signs of disease were present (12 weeks) and finished at the time in which the strain has a mortality rate of 50\% (5 months). Our data shows that Pat A treatment reduces circulating levels of proinflammatory cytokines and autoantibodies increasing survival. An improvement in cognitive functions in treated animals was also observed with neural behaviour tests (learning/memory, and depression) together with a reduction of proinflammatory cytokines in the hippocampus. We did not observe any side effects of the treatment. Alltogether our data suggests that inhibition of translation initiation has an effect in controlling disease activity at the immunological and neurological levels and open a new line of research for strategies to treat lupus and other autoimmune diseases based on the inhibition of translation at early stages.

\section{S6D:7 NOVEL ALPHA-1-ANTITRYPSIN FRAGMENT AS POTENTIAL TREATMENT OF SYSTEMIC LUPUS ERYTHEMATOSUS}

${ }^{1} \mathrm{U}$ Wormser, ${ }^{1} \mathrm{~B}$ Brodsky, ${ }^{2} Y$ Finkelstein, ${ }^{1} \mathrm{E}$ Shapira, ${ }^{1} \mathrm{E}$ Proscura. ${ }^{1}$ The Hebrew University, Jerusalem, Israel; ${ }^{2}$ Shaare Zedek Medical Centre, Jerusalem, Israel

\subsection{6/lupus-2018-abstract.40}

Systemic lupus erythematosus (SLE) is an autoimmune disease characterised by a loss of immunologic tolerance, production of auto-antibodies and inflammatory damage in multiple organs. We have tested the effect of a novel anti-inflammatory peptide, a fragment of alpha-1-antitrypsin, termed UBE on two animal models of SLE, MRL/lpr and NZBW/F1 mice. Treatment of MRL/lpr mice with low dose of UBE (1 micro$\mathrm{gram} / \mathrm{kg}$ ) at early stage of disease namely, 12 weeks old mice, caused significant reduction in proteinuria and hematuria. The beneficial effect of the peptide was corroborated by histological examination. Furthermore, a significant reduction in serum IL17, IL12 and anti dsDNA antibodies was observed in the UBE-treated mice. Isolated CD4 cells incubated with the peptide showed similar cytokine profile. Decreased levels of double negative CD4-CD8- and B220+cells were determined in lymph organs of UBE-treated animals. Similar effects were observed with NZBW/F1 mice, namely, the peptide $(0.3$ microgram $/ \mathrm{kg}$ ) caused significant reduction in proteinuria and, kidney and lung damage as determined in histopathological examination. The highly important SLE serum factor B-lymphocyte stimulator (BLyS or BAFF) was dramatically decreased in UBE-treated mice. Spleen and blood double negative CD4CD8- and serum IL6, IP10 and MCP1 were significantly reduced in peptide-treated mice. The beneficial effects of UBE suggest this peptide as potential drug for SLE. 


\section{S7a - New therapeutic candidates}

\section{S7A:4 REDUCTION OF SYSTEMIC LUPUS FLARES BY ATACICEPT IN A RANDOMISED, PLACEBO- CONTROLLED, PHASE IIB STUDY (ADDRESS II) AND ITS EXTENSION STUDY}

${ }^{1} \mathrm{DJ}$ Wallace, ${ }^{2} \mathrm{DA}$ Isenberg, ${ }^{3} \mathrm{~A}$ Kao, ${ }^{3} \mathrm{C}$ Vazquez-Mateo, ${ }^{3} \mathrm{P}$ Fleuranceau-Morel, ${ }^{3} \mathrm{P}$ Chang, ${ }^{4} \mathrm{JT}$ Merril. 'Cedars-Sinai Medical Centre, University of California Los Angeles, USA; ${ }^{2}$ University College London, UK; ${ }^{3}$ EMD Serono Research and Development Institute, Inc. (a business of Merck KGaA, Darmstadt, Germany), Billerica, USA; ${ }^{4}$ Oklahoma Medical Research Foundation, Oklahoma City, USA

10.1136/lupus-2018-abstract.41
Purpose Atacicept targets the B-cell stimulating factors, BLyS and APRIL, and has shown evidence of clinical response in patients with SLE. The 24 week Phase II ADDRESS II (NCT01972568) Study and its long-term extension (LTE; NCT02070978) provided data on disease activity with up to 48 weeks of atacicept treatment.

Methods In ADDRESS II, patients were randomised (1:1:1) to receive weekly atacicept (75 or $150 \mathrm{mg}$ SC injection) or placebo (PBO) for 24 weeks. Those who completed treatment were eligible to enter the LTE, to either continue on the same atacicept dose (atacicept groups), or switch from PBO to atacicept $150 \mathrm{mg}(\mathrm{PBO} / 150 \mathrm{mg})$. The SLE flare analysis from both studies are reported here.

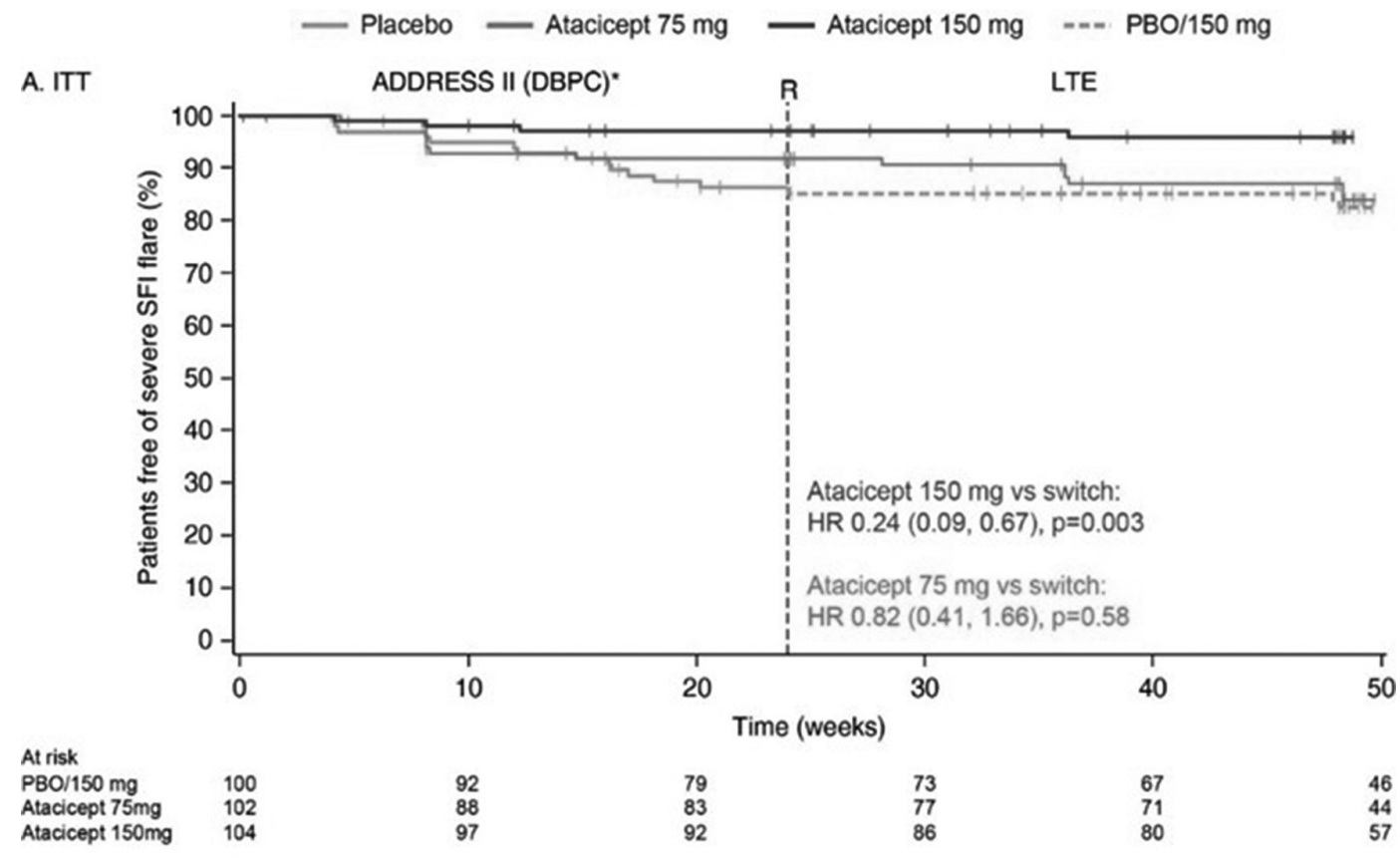

B. HDA

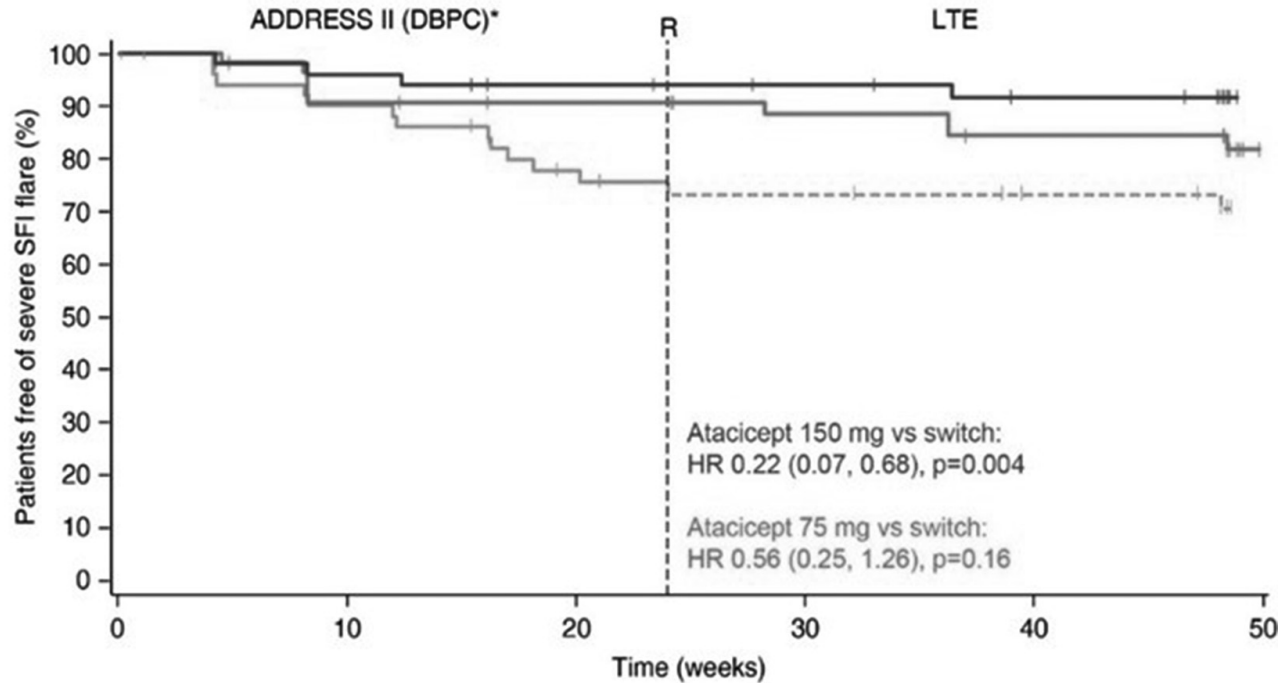

PBO/150 mg Atacicept $75 \mathrm{mg}$ Atacicept $150 \mathrm{mg}$

$\begin{array}{ll}52 & 45 \\ 55 & 48 \\ 51 & 47\end{array}$

36
46
44

32
42
42

$\begin{array}{ll}29 & 21 \\ 39 & 23 \\ 39 & 25\end{array}$

"double-blind placebo-controlled 